\title{
Coupling enhanced sampling of the apo-receptor with template-based ligand conformers selection: performance in pose prediction in the D3R Grand Challenge 4
}

\author{
Andrea Basciu ${ }^{1} \cdot$ Panagiotis I. Koukos ${ }^{2} \cdot$ Giuliano Malloci $^{1}$ (D) - Alexandre M. J. J. Bonvin ${ }^{2}$ (D) Attilio V. Vargiu ${ }^{1,2}$ (D)
}

Received: 10 June 2019 / Accepted: 30 October 2019 / Published online: 13 November 2019

(c) Springer Nature Switzerland AG 2019

\begin{abstract}
We report the performance of our newly introduced Ensemble Docking with Enhanced sampling of pocket Shape (EDES) protocol coupled to a template-based algorithm to generate near-native ligand conformations in the 2019 iteration of the Grand Challenge (GC4) organized by the D3R consortium. Using either AutoDock4.2 or HADDOCK2.2 docking programs (each software in two variants of the protocol) our method generated native-like poses among the top 5 submitted for evaluation for most of the 20 targets with similar performances. The protein selected for GC4 was the human beta-site amyloid precursor protein cleaving enzyme 1 (BACE-1), a transmembrane aspartic-acid protease. We identified at least one pose whose heavy-atoms RMSD was less than $2.5 \AA$ from the native conformation for 16 (80\%) and 17 (85\%) of the 20 targets using AutoDock and HADDOCK, respectively. Dissecting the possible sources of errors revealed that: $(i)$ our EDES protocol (with minor modifications) was able to sample sub-ångstrom conformations for all 20 protein targets, reproducing the correct conformation of the binding site within $\sim 1 \AA$ RMSD; (ii) as already shown by some of us in GC3, even in the presence of near-native protein structures, a proper selection of ligand conformers is crucial for the success of ensemble-docking calculations. Importantly, our approach performed best among the protocols exploiting only structural information of the apo protein to generate conformations of the receptor for ensemble-docking calculations.
\end{abstract}

Keywords Molecular docking · Metadynamics $\cdot$ EDES $\cdot$ HADDOCK $\cdot$ AutoDock $\cdot$ BACE-1

\section{Introduction}

The Drug Design Data Resource (D3R) 2019 Grand Challenge is the fourth iteration (GC4) of the major docking competition organized by the D3R consortium [1-3]. The

Electronic supplementary material The online version of this article (doi:https://doi.org/10.1007/s10822-019-00244-6) contains supplementary material, which is available to authorized users.

Alexandre M. J. J. Bonvin

a.m.j.j.bonvin@uu.nl

$\triangle$ Attilio V. Vargiu

vargiu@dsf.unica.it; a.v.vargiu@uu.nl

1 Dipartimento Di Fisica, Università Di Cagliari, Cittadella Universitaria, S.P. 8 km 0.700, 09042 Monserrato, Italy

2 Bijvoet Center for Biomolecular Research, Faculty of Science - Chemistry, Utrecht University, Padualaan 8, 3584, CH,

Utrecht, The Netherlands competition has two main goals: (i) assessing the ability of docking algorithms to accurately predict the binding poses of a protein against a diverse set of small molecules, and (ii) evaluating of the performance of binding affinity predictors.

The target in this iteration of the pose prediction assessment is the beta-site amyloid precursor protein cleaving enzyme 1 (BACE-1), a beta-secretase 1 protein [4]. BACE-1 plays an early role in Alzheimer's disease, as it is essential for the generation of the $\beta$-amyloid peptides composing the amyloid plaques which are the hallmark neuropathological lesions $[5,6]$. Given its role in initiating the formation of $\beta$-amyloids, BACE- 1 has been a critical target in developing therapies against the progression of Alzheimer's disease [7], as testified also by the huge number of BACE-1 protein structures deposited in the Protein Data Bank (PDB) [8] at the beginning of the challenge ( $>300$ on September 4th 2018). Most of these structures contain putative BACE-1 inhibitors, further witnessing the tremendous potential of this target for treating Alzheimer's disease [9, 10]. 
Here we report the performance of a new approach for ensemble-docking [11, 12], which couples our recently proposed EDES (Ensemble-Docking with Enhanced-sampling of pocket Shape) protocol to sample holo-like and druggable conformations of proteins [13] by means of MD simulations $[14,15]$ with the template-based algorithm for ligand conformer generation successfully employed in the previous GC3 competition [16]. EDES is peculiar in that, regarding the search for holo-like conformations of proteins, it exploits only the experimental structure of the apo-enzyme (PDB ID 1SGZ [17]). The method enhances the sampling of druggable (prone to host ligands) conformations of a given receptor by means of metadynamics [18] simulations. Namely, EDES exploits an original set of collective variables (CVs) describing the geometry (that is, the shape and the volume) of the putative binding site(s). The method has been validated against targets undergoing very minor (single sidechain rearrangement) to very large (hinge-bending motions) conformational changes upon ligand binding [13]. In all cases, EDES was able to sample conformations of the binding site nearly identical to those occurring in the X-ray structures of the reference complexes, as well as to yield top ranked near-native docking poses, thus validating its potential as a new general approach to improve structure-based drug design.

The method for generating ligand structures [16] selects a number (here 10) of ligand conformations out of a pool of (up to 500) conformers generated using the OpenEye Omega software [19] by searching for similar structures on the PDB based on the matching between extended Tanimoto coefficients. As such, it still does require available 3D structures of related ligands bound to the receptor to be present in the PDB. It is however a first step toward fully blind docking from the aporeceptor in cases where no single structure of a related ligand would be present in the PDB.

We benchmark here for the first time in a blind docking experiment our hybrid approach in which proteins conformations obtained from enhanced sampling of the apo structure are combined with ligand conformers selected using the templated-based approach described previously. Near-native ligand poses were found for $16(80 \%)$ and $17(85 \%)$ of the 20 targets using AutoDock [20] and HADDOCK [21, 22], respectively. The most challenging ligands were those whose selected conformers displayed the largest deviation from the true geometry in the native complex. Importantly, our method performed best among those using conformations of the receptor generated without exploiting previous structural information of BACE-1 in complex with other ligands.

\section{Materials and methods}

The D3R GC4 is divided into a set of different stages, in which the participants are requested to predict the binding pose of a set of different ligands against the same receptor and to rank them and/or estimate their free energies of binding. We participated in stages $1 \mathrm{a}$ and $1 \mathrm{~b}$ of the pose prediction challenge. Specifically, in stage 1a (cross-docking) we performed ensemble docking calculations on conformations of the protein and of the ligands generated by our methodology (vide infra), while in stage $1 b$ (self-docking) the 10 conformers of each ligand were docked onto the conformations of BACE-1 extracted from the structures of their complexes with the 20 compounds in the dataset. For both challenges the participants were asked to generate a ranked set of maximum 5 poses for each ligand.

\section{Data provided}

In stage $1 \mathrm{a}$ the only data provided by the organizers consisted of a list of 20 SMILES entries (corresponding to the 20 compounds for which the participants were asked to predict the crystallographic pose) and of the protein sequence in FASTA format. In stage $1 \mathrm{~b}$ the experimental structures of the receptors for all 20 BACE-1 complexes were provided, to allow the participants to re-dock each ligand on the corresponding holo-conformation of the receptor.

\section{Binding site determination}

In order to identify the putative binding site of the protein, we used the same approach presented in [16]. Namely, we retrieved in the PDB all the structures featuring at least $95 \%$ sequence identity to the amino acid sequence provided by the organizers and having a co-crystallized ligand (other than crystallization buffer molecules); this resulted in 340 entries. We verified that the binding site was well characterized and perfectly conserved in all the structures with no missing residues in the putative pocket. Next, we used the Tanimoto coefficient, as implemented in fmcsR [24] and chemmineR [25] packages to evaluate the similarity between the ligands present in the 340 entries and each of the 20 compounds provided, in order to identify a set of receptor templates featuring the most similar ligand to the compounds to be docked. Details on Tanimoto similarity measurement can be found in $[16,26]$. The search for the structure featuring the most similar ligand to each of the 20 compounds resulted in 10 complex structures selected as templates ((PDB IDs: 2B8L, 2IQG, 3DV1, 3DV5, 3K5C, 3VEU, 4DPI, 4KE1, 4R92, 6BFD; see Table S1). In addition to providing a metric to select ligands conformers for 
docking calculations (vide infra), these structures were used to identify the residues lining the putative binding site. This list was built by merging all the residues within $3.5 \AA$ from the ligand in each of the 10 complex structures selected. Since this approach generated a very large number of residues (more than 30), we kept only the most conserved ones (appearing at least in two structures) and, among those present only in one structure, the most buried ones (likely to interfere with ligand binding). The resulting selection is shown in Fig. 1, which clearly highlights that the chosen list of 20 residues (see Table S2) surrounds all the 20 congeneric ligands provided for this challenge.

\section{Ligand preparation}

The 20 ligands were similar in size, each containing about 35 heavy atoms. For the generation of their conformers we employed the methodology featured in [16]. The protocol makes use of ligand similarities in the form of Tanimoto coefficients and can be summarized in the following steps (the first three steps are the same ones performed to identify the BS and resulted in the ten template structures reported in Table S1):

1. Identify existing highly homologous structures of the target protein bound to small molecules;

2. Discard undesirable structures (e.g. low resolution, split side chains near the binding site, covalently bound ligands in the case where the target ligand is known to bind non-covalently, etc.);

3. Calculate Tanimoto coefficients between all target and template ligands;

4. Generate up to 500 conformers for all ligand targets using the OpenEye OMEGA software package [19];

5. Select 10 conformers for all targets by comparing the generated conformers to the structure of the template ligand with the highest Tanimoto similarity per ligand, with OpenEye ROCS (shape and colour mode) [23].

\section{Unbiased and enhanced-sampling molecular dynamics (MD) simulations}

In this work we applied essentially the same workflow presented in the original EDES publication (see [13] for details), with some minor modifications that are described in the following sections.

\section{Starting protein structure}

We used the package BLASTP 2.7.1+[27] to search for protein structures homologous to the sequence provided by the organizers. We searched the PDB, setting the number of alignments (-num_alignments) to 1000 and the number of
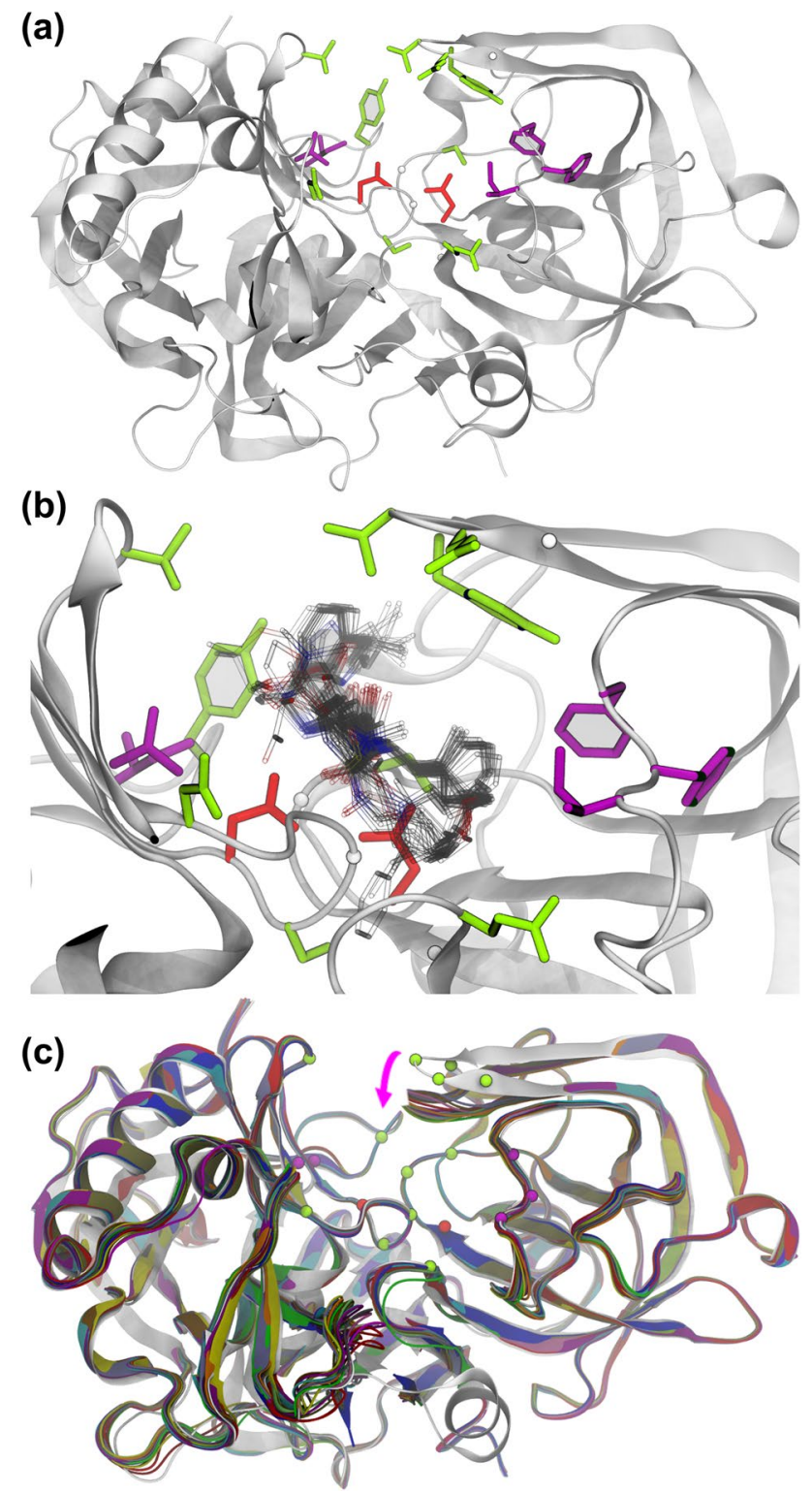

Fig. 1 Putative binding site identified on the BACE-1 apo protein (PDB ID 1SGZ [17]) for implementation of the EDES approach. a Structure of the protein (grey ribbons) showing the sidechains of the 20 residues in Table 2 as sticks colored by type (polar, apolar, acidic and glycines in light green, magenta, red and white respectively); b zoom on the putative binding site in a), showing in transparent sticks the experimental poses of the 20 ligands provided by the organizers (after superposition of common $\mathrm{C}_{\alpha}$ atoms on all proteins to $1 \mathrm{SGZ}$ ); $\mathbf{c}$ comparison between the apo structure of BACE-1 (grey ribbons) and the 20 ligand/BACE-1 complex structures (blue ribbons) released at stage $1 \mathrm{~b}$ of the challenge. The magenta arrow highlights the major displacement undergone by the protein flap upon ligand binding

scoring evaluations (-evalue) to 10, while using the default values otherwise. We also requested the structures to have the word "BACE" in their name. With these search criteria, we identified around 300 structures, which included only eight apo proteins. Among these, we identified as template 
the structure with PDB ID 1SGZ [17], which was resolved at good resolution ( $2 \AA$ ), did not feature any missing residue, and displayed a full overlap with the BACE-1 sequence provided by the organizers. Prior to setting up the system, the template structure was further refined through the MolProbity webserver [28].

\section{Unbiased MD}

Standard all-atom MD simulations of the apo protein (herafter $\left.\mathrm{MD}_{\text {apo }}\right)$ embedded in a $0.15 \mathrm{KCl}$ water solution ( 60.000 atoms in total) and under periodic boundary conditions were carried out using the pmemd module of the AMBER18 package [29]. The initial distance between the protein and the edge of the box was set to be at least $16 \AA$ in each direction. Topology files were created for each system using the $L E a P$ module of AmberTools 18 starting from the apo structure with PDB ID 1SGZ. The AMBER-FB15 $[30,31]$ force field was used for the protein, the TIP3P-FB model was used for water, and the parameters for the ions were obtained from [32]. Long-range electrostatics was evaluated through the particle-mesh Ewald algorithm using a real-space cutoff of $12 \AA$ and a grid spacing of $1 \AA$ in each dimension. The van der Waals interactions were treated by a Lennard-Jones potential using a smooth cutoff (switching radius $10 \AA$, cutoff radius $12 \AA$ ). Multistep energy minimization with a combination of the steepest-descent and conjugate-gradient methods was carried out to relax internal constraints of the systems by gradually releasing positional restraints. Following this, the system was heated from 0 to $310 \mathrm{~K}$ in $10 \mathrm{~ns}$ of constant-pressure heating (NPT) using the Langevin thermostat (collision frequency of $1 \mathrm{ps}^{-1}$ ) and the Berendsen barostat. After equilibration, a production run of $1 \mu \mathrm{s}$ was performed. A time step of $2 \mathrm{fs}$ was used for preproduction runs, while equilibrium MD simulations were carried out with a time step of $4 \mathrm{fs}$ in the NPT ensemble (using a MC barostat) after hydrogen mass repartitioning [33]. Coordinates from production trajectory were saved every $100 \mathrm{ps}$.

\section{Enhanced sampling MD}

EDES aims to generate holo-like conformations of a protein exploiting only structural information on the apo counterpart. This is achieved by means of bias-exchange well-tempered metadynamics simulations $[34,35]$ on a set of generic CVs effectively biasing both the shape and the volume of the binding pocket. Ultimately, the method mimics induced fit rearrangements of the receptor due to ligand/protein interactions. Metadynamics simulations were performed on the apo protein using the GROMACS 2016.5 package [36] and the PLUMED 2.3.5 plugin [37]. Simulations were started from the last conformation sampled along the pre-production step of the unbiased MD. AMBER parameters were ported to GROMACS using the acpype parser [38]. Following the original implementation, four CVs defined considering only protein residues within the binding site were used: (1) the radius of gyration of the binding site (hereafter $\mathrm{RoG}_{\mathrm{BS}}$ ) calculated using the gyration built-in function of PLUMED; $(2-4)$ the numbers of (pseudo)contacts across three orthogonal "inertia planes" (CIPs), calculated through a switching function implemented in the coordination keyword of PLUMED. The inertia planes are defined as the planes orthogonal to the three principal inertia axes of the binding site and passing through its geometrical center. All non-hydrogenous atoms were considered to define the three CIPs, while only backbone atoms were used to estimate $\mathrm{RoG}_{\mathrm{BS}}$, on which we also implemented a "windows" approach aimed to sample in a controlled manner different shapes of the binding site. Namely, we applied soft restraints at $\mathrm{RoG}_{\mathrm{BS}}$ values that are $7.5 \%$ higher and lower than the $\mathrm{RoG}_{\mathrm{BS}}$ of the X-ray apo structure $\left(\mathrm{RoG}_{\mathrm{X} \text {-ray }}{ }^{\mathrm{apo}}\right)$ and from that trajectory, corresponding to the first window, we randomly selected a conformation associated with a $\mathrm{RoG}_{\mathrm{BS}}$ value $5 \%$ lower than $\mathrm{RoG}_{\mathrm{X} \text {-ray }}$ apo. This structure was used as starting point for another MD simulation (corresponding to window 2) with walls centered at $\pm 7.5 \% \mathrm{RoG}_{\mathrm{X} \text {-ray }}{ }^{\text {apo }}$ from this new center. We repeated the procedure until we generated three windows including the first one, centered at 9.91, 9.41, and $8.92 \AA$ respectively (corresponding to a $\sim 10 \%$ decrease of $\mathrm{RoG}_{\mathrm{X} \text {-ray }}{ }^{\text {apo }}$ ). Note that the choice of biasing the conformational sampling towards structures corresponding to values of $R_{0 G} G_{B S}$ lower than $R_{0} G_{\text {X-ray }}$ apo, primarily due to our intention to validate the original implementation of the protocol, is justified a posteriori by the (small) collapse of the binding site of BACE- 1 occurring upon binding of all ligands (see "Results and discussion"). More generally, several studies showed that the binding of ligands to enzymes most often stabilizes a closed conformation of their binding pockets as compared to the apo structures (see e.g. [39, 40].).

Each replica was simulated for $100 \mathrm{~ns}$, leading to $400 \mathrm{~ns}$ of metadynamics simulations per window; coordinates were saved every $10 \mathrm{ps}$. Note that in [13] we demonstrated that EDES is not sensitive to the exact choice of the windows parameters. The height $w$ of the Gaussian hills was set to $0.6 \mathrm{kcal} / \mathrm{mol}$, while the widths $s_{i}$ of the Gaussian hills were set to $0.06,2.6,1.7$ and 3.0 respectively for $R_{0} G_{B S}$ and $\mathrm{CIP}_{1,2,3}$, respectively. The bias factor for well-tempered metadynamics was set to 10 . Hills were added every 2 ps, while the bias-exchange frequency was set to $20 \mathrm{ps}$. The force constants for the restraints on the $\mathrm{RoG}_{\mathrm{BS}}$ were set to 50 and $10 \mathrm{kcal} \mathrm{mol}^{-1} \AA^{-2}$ for the upper and lower walls respectively. Hereafter, we will refer to these simulations as $\operatorname{EDES}_{3 \mathrm{w}}$. 


\section{Docking}

Ensemble docking calculations were performed using either AutoDock4.2 [20] or the HADDOCK2.2 webserver $[21,22]$, following the same procedures described in [13]. However, at odd with the original implementation, calculations were here performed on 200 protein conformations obtained by merging structures extracted from both $\mathrm{MD}_{\text {apo }}$ and $\operatorname{EDES}_{3 \mathrm{w}}$ simulations (see Fig. S1). This variant of the original approach appeared to be reasonable, as the extent of the conformational changes occurring at the binding site was unknown a priori. In particular, since we expected to observe significant oscillations of this region including the flap (Fig. 1) also along $\mathrm{MD}_{\text {apo, }}$, including structures extracted from that trajectory should encompass a non-negligible fraction of structures with $\mathrm{RoG}_{\mathrm{BS}}$ larger than the value calculated on 1SGZ. In addition, the number of protein conformations was lowered to 200 to cope with the time constraints of the challenge, also considering that for each compound, ten ligand conformations were employed in ensemble-docking runs. Protein conformations were extracted by means of a multi-step cluster analysis as described in [13], with the additional requirement to extract at least ten cluster representatives from each of the ten slices in which the $\mathrm{RoG}_{\mathrm{BS}}$ distributions were binned, so as to include a certain number of structures also from poorly sampled regions. The multistep cluster analysis was applied separately to $\mathrm{MD}_{\text {apo }}$ and $\mathrm{EDES}_{3 \mathrm{w}}$, extracting 500 clusters from each trajectory. Next, an additional cluster analysis using the same approach was performed on the pool of 1000 cluster representatives in order to generate the final ensemble of 200 conformations. For AutoDock, the docking was repeated with each selected conformer, while for HADDOCK the ensemble of conformers was submitted and used in a single docking run. We submitted four sets of protein-ligand binding pose predictions generated through the following docking protocols:

1. Autodock (receipt ID pe6zg): Docking calculations and pose selection were performed following prescriptions detailed for Autodock in [13]. Briefly, for each ligand, 10 different conformers were docked on each of the 200 protein structures using the Lamarckian Genetic Algorithm (LGA). In order to cope with the schedule of the Challenge, the ligand conformers were kept rigid during docking (ensuring a correct cycle connectivity). The grid density and number of energy evaluations were both increased from default values (respectively by decreasing the spacing parameter from 0.375 to $0.25 \AA$ and by increasing the ga_num_evals parameter by a factor of 10 ) in order to avoid repeating each calculation several times to obtain converged results. An adaptive grid was used, enclosing all of the residues belonging to the binding site in each different protein conformation. Next, the top poses (in total 200, one for each docking run) were clustered using the cpptraj module of AmberTools18 with a hierarchical agglomerative algorithm. Namely, after structural alignment of the protein binding site conformations, the poses were clustered using a distance RMSD (dRMSD) cutoff $d_{\mathrm{c}}=0.075 \cdot N_{\mathrm{nh}}$, where $N_{\mathrm{nh}}$ is the number of non-hydrogenous atoms of the ligand. This choice was made in order to tune the cutoff to the molecular size of each compound. Finally, clusters were ordered according to the top score (lowest binding free energy) within each cluster.

2. HADDOCK (receipt ID kmtri): Docking calculations and pose selection were performed following prescriptions detailed for HADDOCK in [13]. A single docking run was performed per case, starting from the various ensembles of 200 conformations, with increased sampling (10,000/400/400 models for it 0 , itl and wat steps, respectively referring to rigid-body docking, semiflexible and final refinement in explicit solvent). Namely, during it 0 the protein binding site residues were defined as "active", effectively drawing the rigid ligand into the binding site without restraining its orientation. For the subsequent stages only the ligand was active, improving its exploration of the binding site while maintaining at least one contact with its residues. During these stages the cycle connectivity was maintained owing to the definition of bonding networks and parameters through PRODRG [41], as customary implemented in the HADDOCK webserver. The weight of the intermolecular van der Waals energy used in it 0 was increased to 1.0 (from the default value of 0.01), and RMSD-based clustering was selected with a cutoff of $1 \AA$. Docking was guided by ambiguous distance restraints defined for the residues of the binding site and the ligand.

3. Autodock with pose refinement and rescoring (herafter Autodock ${ }_{r r}$; receipt ID nstab): This approach is the same as in 1, with an additional step consisting in the relaxation of the top 10 docking poses by means of a multi-step optimization performed with AMBER18 [29]. Namely, the systems were optimized in vacuum through three consecutive cycles of restrained structural relaxation (1000 cycles of steepest descent followed by up to 24,000 cycles of conjugate gradients) followed by an unrestrained optimization (2000 cycles of steepest descent followed by up to 8000 cycles of conjugate gradients). During restrained relaxation harmonic forces of $0.3,0.2$, and $0.1 \mathrm{kcal} \mathrm{mol}^{-1} \AA^{-1}$ respectively for the first, second and third cycles were applied on all nonhydrogenous atoms of the system. Long-range electrostatics was evaluated directly using a cutoff of $99 \AA$, as for the Lennard-Jones potential. The AMBER-FB15 $[30,31]$ force field was used for the protein, while the parameters of the ligands were derived from the GAFF 
force field [42] using the antechamber module of AmberTools18. In particular, bond-charge corrections (bcc) charges were assigned to ligand atoms following structural relaxation under the Austin Model 1 (AM1) approximation. Note that, as for HADDOCK, the definition of a topology involving permanent bonding interactions allows for keeping the correct cycle connectivity during refinement, while including some flexibility (e.g. by moving torsional angles and permitting the formation of H-bonds). Finally, the poses were rescored using the same scoring function of AutoDock employed to rank the original docking poses.

4. HADDOCK $K_{\text {all-Hs }}$ (receipt ID apue7): this approach is the same as in 2, except for the inclusion of all hydrogens (and not only the polar ones) in atomic models.

In addition to ensemble-docking calculations using receptor structures generated in silico, during stage $1 \mathrm{~b}$ we also performed self-docking calculations (only with AutoDock), using for each ligand the conformation of the receptor extracted from the corresponding holo experimental structure released by the organizers at the beginning of stage $1 \mathrm{~b}$ (protocol Autodock self, receipt ID qb4hg). All the remaining parameters were identical to those employed within the Autodock protocol.

Note that we also submitted predictions using the same template-based protocol described in [16] $\left(H A D D O C K_{t b}\right.$; receipt ID $n w m 5 a$ ). Those led to the best performance from all our submissions but will not be discussed here.

\section{Druggability calculations}

Following our previous study [13] we used the package f-pocket [43] to assess the druggability of the binding site within the ensembles of BACE-1 conformations generated by $\mathrm{MD}$ simulations. For each conformation, we evaluated the druggability score $D$ [44] ranging from 0 to 1 with higher values identifying more druggable geometries. It is customary to associate scores $>0.5$ to putative binding sites [44].

\section{Results and discussion}

In the following we report the performance of our protocols in predicting near-native binding poses of BACE-1 ligands (stage 1a). Next we report the performance of Autodock in self-docking calculations of the same ligands onto the experimental structure of the receptor released at stage $1 \mathrm{~b}$. Evaluations were performed according to the data downloaded from the D3R website (https://drugdesigndata.org/ about/grand-challenge-4-evaluation-results). The accuracy of the poses was evaluated by calculating the RMSD of each ligand with respect to its experimental reference structure (considering only heavy atoms), after superposition of the binding interface areas. First, we discuss the performance in terms of both global and per ligand descriptors. Next, to identify possible sources of errors, we analyze the accuracy of our EDES-like approach in sampling holo-like and druggable conformations of BACE-1, and the performance of the template-based algorithm to generate accurate ligand conformers [16]. We conclude by summarizing the possible drawbacks of the methodology and its unique features, and by listing possible routes of future development.

\section{Stage 1a}

Table 1 provides an overview of the performance of our methodologies in finding near-native poses of the 20 BACE-1 ligands. The best results were obtained with AutoDock [20] coupled to a multi-step structural optimization and rescoring of the top 10 poses (Autodock $k_{r r}$ ). Using this approach, we found median and average values of the RMSD calculated on the top pose of each ligand (hereafter $\mathrm{RMSD}_{\text {med }}^{1}$ and $\langle\mathrm{RMSD}\rangle^{1}$ ) lower than $2 \AA$ and $3 \AA$, respectively. Moreover, these values were lower than $1.5 \AA$ and $2 \AA$ when calculated on the nearest-native poses (hereafter $\mathrm{RMSD}_{\text {med }}^{\mathrm{min}}$ and $\left\langle\mathrm{RMSD}^{\mathrm{min}}\right\rangle$, respectively). The AMBERbased refinement led to significant improvements with respect to the "standard" Autodock protocol, for which we
Table 1 Overall performance of our protocols in retrieving nearnative ligands conformations of BACE-1 ligands (rows 4-8) during stage 1a, and performance of the Autodock self $_{\text {s }}$ protocol in stage $1 \mathrm{~b}$ (last row; data from https://drugdesign data.org)

\begin{tabular}{|c|c|c|c|c|c|c|}
\hline \multirow[t]{2}{*}{ Protocol } & \multicolumn{3}{|l|}{ Averages } & \multicolumn{3}{|l|}{ Median } \\
\hline & $\left\langle\mathrm{RMSD}^{\mathrm{min}}\right\rangle$ & $\left\langle\mathrm{RMSD}^{1}\right\rangle$ & $\langle$ RMSD $\rangle$ & $\mathrm{RMSD}_{\text {med }}^{\min }$ & $\mathrm{RMSD}_{\text {med }}^{1}$ & $\mathrm{RMSD}_{\text {med }}$ \\
\hline \multicolumn{7}{|l|}{ Stage 1a } \\
\hline Autodock $_{r r}$ & $1.73 \pm 0.88$ & $2.86 \pm 2.71$ & $4.24 \pm 1.77$ & 1.38 & 1.78 & 3.89 \\
\hline Autodock & $2.48 \pm 1.82$ & $3.10 \pm 2.57$ & $4.41 \pm 2.10$ & 2.07 & 2.25 & 4.28 \\
\hline HADDOCK & $2.28 \pm 0.99$ & $4.12 \pm 2.73$ & $4.64 \pm 1.53$ & 2.06 & 3.12 & 4.23 \\
\hline$H A D D O C K_{\text {all-Hs }}$ & $3.19 \pm 2.26$ & $4.83 \pm 3.50$ & $5.96 \pm 2.18$ & 2.66 & 3.10 & 5.76 \\
\hline $\begin{array}{l}\text { Autodock } \\
\text { Stage } 1 \mathrm{~b}\end{array}$ & $3.78 \pm 2.94$ & $5.67 \pm 3.72$ & $5.17 \pm 3.34$ & 2.47 & 3.51 & 3.49 \\
\hline Autodock $k_{\text {self }}$ & $2.24 \pm 2.13$ & $2.93 \pm 2.78$ & $3.59 \pm 2.73$ & 1.60 & 2.03 & 2.30 \\
\hline
\end{tabular}

All values are expressed in $\AA$ 
obtained values of $\mathrm{RMSD}_{\text {med }}^{\mathrm{min}}$ and $\left\langle\mathrm{RMSD}^{\mathrm{min}}\right\rangle$ lower than $2.5 \AA$. A very similar performance was achieved by HADDOCK when using standard settings, while the explicit consideration of non-polar hydrogen atoms of the ligand during docking led to an appreciable drop in the accuracy. While a clear-cut explanation of this behavior would require a more systematic study that is out of the scope of this manuscript, it seems reasonable that the tiny Van der Waals volumes of nonpolar hydrogens have a large impact on the ability of HADDOCK to place the ligands within the buried binding site of BACE-1.

The $\left\langle\mathrm{RMSD}^{\mathrm{min}}\right\rangle$ and $\left\langle\mathrm{RMSD}^{1}\right\rangle$ metrics place our methods in the middle-left and middle-right regions of the histogram plot summarizing performances of all applicants (Fig. 2). An inspection of the protocols employed in this competition that performed better than any of ours (in terms of $\left\langle\mathrm{RMSD}^{1}\right\rangle$ ) revealed that our methodology is (among those for which these details were disclosed) the only one based on ensemble-docking calculations using protein conformations generated in silico starting from an apo experimental structure of BACE-1. Thus, no information on the structures of its complexes with similar ligands was exploited to bias the conformation of the binding site towards holo-like geometries. In order to investigate more in detail the performance of our method, and possibly to correlate the accuracy of our results to one or more relevant parameters, we report the results for each of the 20 BACE ligands in Table 2, as well as in Fig. 3a-d for Autodock, Autodock ${ }_{r r}$, HADDOCK, and $H A D D O C K_{\text {all-Hs }}$, respectively. The Table reveals that the aforementioned approaches gave at least one pose with $\mathrm{RMSD}_{\text {lig }}<2.5 \AA$ respectively in $15,16,17$, and 10 out of 20 cases, corresponding to success rates of $75 \%, 80 \%, 85 \%$, and $50 \%$.

In the following, we will discuss in more detail only the top three approaches: Autodock, Autodock $r$, and HADDOCK. Inspection of Table 2 reveals that the most challenging ligand was $\mathrm{BACE}_{02}$, which is the only compound for which we obtained poses featuring an RMSD $>3 \AA$ from the native conformation with all approaches. Additional challenging ligands include $\mathrm{BACE}_{10}$, for which the best RMSD value was $2.8 \AA$ (obtained with Autodock ${ }_{r r}$ ), and to a minor extent $\mathrm{BACE}_{07}, \mathrm{BACE}_{09}, \mathrm{BACE}_{14}, \mathrm{BACE}_{16}$, and $\mathrm{BACE}_{18}$, for which one out of the three protocols was unable to find poses with RMSD values lower than $2.5 \AA$.
Fig. 2 Overall performance of the protocols employed in this study, as measured by the values of $\left\langle\mathrm{RMSD}^{\mathrm{min}}\right\rangle$ and $\left\langle\mathrm{RMSD}^{1}\right\rangle$
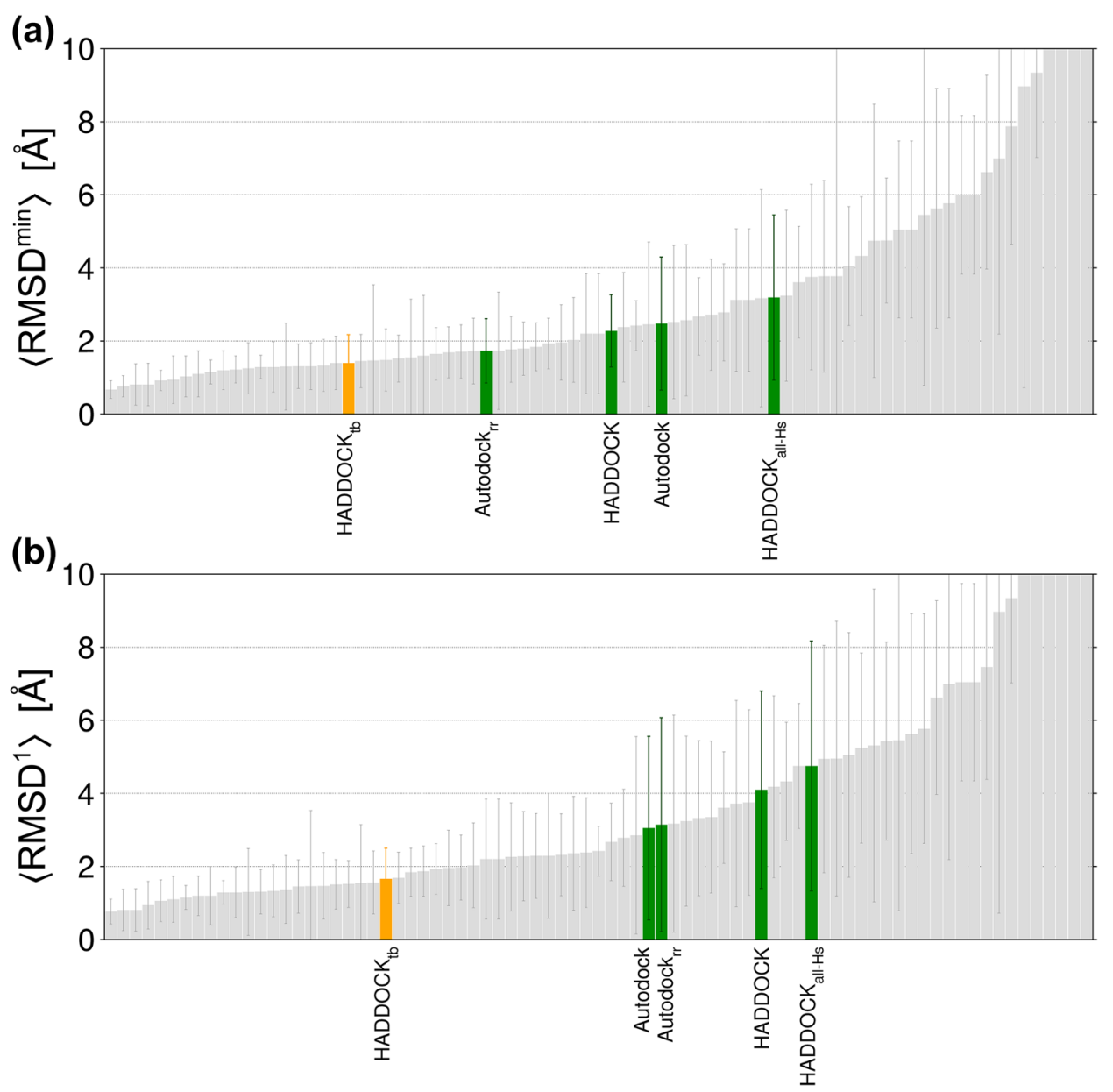
Table 2 Summary of the docking results obtained with the four methods described in this work for each of the 20 BACE-1 ligands (data from https://drugdesigndata.org)

\begin{tabular}{|c|c|c|c|c|c|c|c|c|c|c|c|c|}
\hline \multirow[t]{2}{*}{ Target ligand } & \multicolumn{3}{|l|}{ Autodock } & \multicolumn{3}{|l|}{ Autodock $_{r r}$} & \multicolumn{3}{|c|}{ HADDOCK } & \multicolumn{3}{|c|}{$H A D D O C K_{\text {all-Hs }}$} \\
\hline & $\mathrm{RMSD}^{\min }$ & $\mathrm{RMSD}^{1}$ & $\langle\mathrm{RMSD}\rangle$ & $\mathrm{RMSD}^{\mathrm{min}}$ & $\mathrm{RMSD}^{1}$ & $\langle\mathrm{RMSD}\rangle$ & $\mathrm{RMSD}^{\min }$ & $\mathrm{RMSD}^{1}$ & $\langle\mathrm{RMSD}\rangle$ & $\mathrm{RMSD}^{\min }$ & $\mathrm{RMSD}^{1}$ & $\langle\mathrm{RMSD}\rangle$ \\
\hline $\mathrm{BACE}_{01}$ & 1.7 & 2.2 & $3.7 \pm 2.9$ & 1.2 & 1.2 & $1.8 \pm 0.5$ & 1.5 & 2.0 & $2.8 \pm 1.7$ & 3.1 & 3.1 & $4.7 \pm 2.7$ \\
\hline $\mathrm{BACE}_{02}$ & 4.2 & 4.2 & $6.5 \pm 2.7$ & 4.5 & 4.5 & $7.9 \pm 2.9$ & 3.9 & 4.4 & $4.3 \pm 0.3$ & 10.2 & 10.5 & $11.0 \pm 0.7$ \\
\hline $\mathrm{BACE}_{03}$ & 2.8 & 2.8 & $5.3 \pm 3.4$ & 1.8 & 2.6 & $3.8 \pm 3.4$ & 2.5 & 3.5 & $4.2 \pm 2.7$ & 3.2 & 3.5 & $5.7 \pm 3.2$ \\
\hline $\mathrm{BACE}_{04}$ & 1.5 & 1.5 & $2.9 \pm 1.3$ & 1.1 & 1.1 & $2.2 \pm 1.0$ & 2.2 & 9.6 & $6.6 \pm 4.0$ & 2.3 & 9.7 & $7.1 \pm 3.6$ \\
\hline $\mathrm{BACE}_{05}$ & 2.1 & 2.1 & $3.8 \pm 3.3$ & 1.4 & 1.6 & $5.3 \pm 4.9$ & 1.4 & 3.1 & $3.8 \pm 3.1$ & 8.7 & 9.8 & $10.0 \pm 0.9$ \\
\hline $\mathrm{BACE}_{06}$ & 1.1 & 1.1 & $1.7 \pm 0.7$ & 1.5 & 1.6 & $2.1 \pm 0.8$ & 1.8 & 2.3 & $3.6 \pm 3.3$ & 2.3 & 2.3 & $5.8 \pm 4.6$ \\
\hline $\mathrm{BACE}_{07}$ & 2.1 & 2.5 & $2.5 \pm 0.3$ & 2.6 & 2.6 & $3.4 \pm 0.9$ & 2.4 & 2.6 & $4.5 \pm 3.2$ & 3.5 & 3.6 & $6.2 \pm 3.5$ \\
\hline $\mathrm{BACE}_{08}$ & 1.2 & 1.7 & $3.3 \pm 4.0$ & 1.0 & 1.0 & $4.9 \pm 5.0$ & 1.3 & 1.6 & $2.0 \pm 1.1$ & 1.4 & 1.5 & $5.0 \pm 4.7$ \\
\hline $\mathrm{BACE}_{09}$ & 2.2 & 2.2 & $4.5 \pm 2.9$ & 2.4 & 3.1 & $4.1 \pm 3.1$ & 3.3 & 10.0 & $8.4 \pm 3.0$ & 2.2 & 2.9 & $5.3 \pm 3.8$ \\
\hline BACE $_{10}$ & 9.3 & 9.4 & $9.4 \pm 0.1$ & 2.8 & 10.0 & $8.2 \pm 3.0$ & 5.5 & 5.8 & $6.6 \pm 2.0$ & 3.5 & 9.5 & $6.5 \pm 2.9$ \\
\hline $\mathrm{BACE}_{11}$ & 1.5 & 2.8 & $2.3 \pm 0.8$ & 1.0 & 1.2 & $3.6 \pm 4.3$ & 2.0 & 4.5 & $3.7 \pm 1.0$ & 1.7 & 1.7 & $5.4 \pm 3.9$ \\
\hline $\mathrm{BACE}_{12}$ & 1.4 & 1.5 & $1.8 \pm 0.5$ & 1.2 & 10.3 & $6.8 \pm 4.7$ & 1.2 & 1.9 & $3.5 \pm 3.4$ & 1.4 & 1.9 & $4.8 \pm 4.3$ \\
\hline $\mathrm{BACE}_{13}$ & 1.5 & 2.2 & $2.1 \pm 0.5$ & 0.9 & 1.4 & $3.6 \pm 4.2$ & 1.5 & 1.5 & $3.9 \pm 3.2$ & 1.8 & 1.8 & $2.2 \pm 0.4$ \\
\hline $\mathrm{BACE}_{14}$ & 4.2 & 10.8 & $8.2 \pm 3.6$ & 1.3 & 2.5 & $4.9 \pm 3.3$ & 2.1 & 9.4 & $6.9 \pm 3.8$ & 2.9 & 3.1 & $4.7 \pm 3.5$ \\
\hline BACE $_{15}$ & 2.2 & 2.2 & $4.4 \pm 3.8$ & 1.1 & 1.8 & $2.4 \pm 1.0$ & 2.2 & 3.0 & $3.7 \pm 1.6$ & 3.4 & 9.4 & $8.5 \pm 2.9$ \\
\hline $\mathrm{BACE}_{16}$ & 2.5 & 2.5 & $4.2 \pm 3.6$ & 2.6 & 2.6 & $4.4 \pm 2.9$ & 2.5 & 3.1 & $4.5 \pm 3.5$ & 3.0 & 3.3 & $6.0 \pm 4.0$ \\
\hline $\mathrm{BACE}_{17}$ & 1.8 & 1.9 & $4.8 \pm 2.7$ & 1.4 & 1.7 & $3.1 \pm 2.4$ & 1.9 & 5.7 & $5.2 \pm 1.9$ & 1.7 & 2.3 & $3.0 \pm 1.9$ \\
\hline $\mathrm{BACE}_{18}$ & 2.6 & 2.6 & $5.3 \pm 2.5$ & 1.8 & 1.8 & $4.7 \pm 2.5$ & 2.0 & 3.9 & $4.0 \pm 1.4$ & 1.9 & 2.4 & $3.5 \pm 2.0$ \\
\hline BACE $_{19}$ & 1.8 & 2.3 & $6.8 \pm 4.4$ & 1.3 & 1.5 & $3.4 \pm 3.0$ & 2.3 & 2.3 & $5.1 \pm 3.3$ & 2.4 & 9.6 & $8.1 \pm 3.2$ \\
\hline $\mathrm{BACE}_{20}$ & 2.1 & 2.1 & $4.9 \pm 3.4$ & 1.6 & 8.5 & $4.0 \pm 3.0$ & 2.0 & 2.0 & $5.3 \pm 4.0$ & 3.2 & 3.2 & $5.9 \pm 3.6$ \\
\hline
\end{tabular}

All values are expressed in $\AA$. RMSD ${ }^{\text {min }}$ values larger than $2.5 \AA$ are bolded

Inspection of Fig. 3a-d reveals the large variability in the orientation of docking poses for almost all of the ligands investigated. Such behavior, resulting in several poses displaying large RMSD values and in high standard deviations of the averages, was somewhat expected because of the (desired) tendency of our protocol to maximize the conformational diversity of the binding site of the protein structures used in docking calculations (vide infra). Clearly, considering the average RMSD over all poses would intrinsically penalize approaches like ours. Nonetheless, the best overall performing method (Autodock $k_{r r}$ ) demonstrated its ability to reproduce at least one near-native pose among the top 5 for virtually all ligands but $\mathrm{BACE}_{02}$. In addition to using an ensemble of receptor structures, allowing some degree of flexibility of the ligands (e.g. by activating torsional angles in AutoDock) could in principle improve results for this and the most challenging cases, although improvements were reported to be system-dependent $[45,46]$. This is confirmed by the comparison with results obtained using HADDOCK, which includes by default flexibility of both docking partners by means of short MD runs in the space of the torsional angles.

Finally, we compared our predictions with docking calculations performed with Autodock on the experimental apo structure of BACE-1 (hereafter Autodock apo $_{\text {) }}$. Table 1 confirms that, as expected, the lack of inclusion of protein flexibility has a major impact on the accuracy of near-native pose predictions [45-47]. In a further effort to identify most likely sources of errors we assessed the accuracies of our protocols in sampling near-native conformations of the protein and the ligands prior to docking calculations.

\section{Sampling of holo-like (and druggable) conformations of BACE-1}

The ability of our enhanced-sampling protocol to generate holo-like conformations of BACE-1 was evaluated in terms of the distributions of the RMSD calculated for the non-hydrogenous atoms of the binding site (hereafter $\mathrm{RMSD}_{\mathrm{BS}}$ ) with respect to each of the $20 \mathrm{BACE}-1$ experimental structures (provided at stage $1 \mathrm{~b}$ ) for $\mathrm{MD}_{\mathrm{apo}}, \mathrm{EDES}_{3 \mathrm{w}}$ and the ensemble of 200 cluster structures used in docking calculations.

Figure 4 shows that both $\mathrm{MD}_{\text {apo }}$ and, to a larger extent, EDES $_{3 \mathrm{w}}$, were able to generate a significant fraction of receptor conformations displaying $\mathrm{RMSD}_{\mathrm{BS}}$ values lower than $2 \AA$ with respect to every ligand/BACE-1 experimental structure. In particular, $\mathrm{EDES}_{3 \mathrm{w}}$ performs better than $\mathrm{MD}_{\text {apo }}$ in generating holo-like structures, as testified by the sizeable shoulder seen in the middle panel of Fig. 4, 


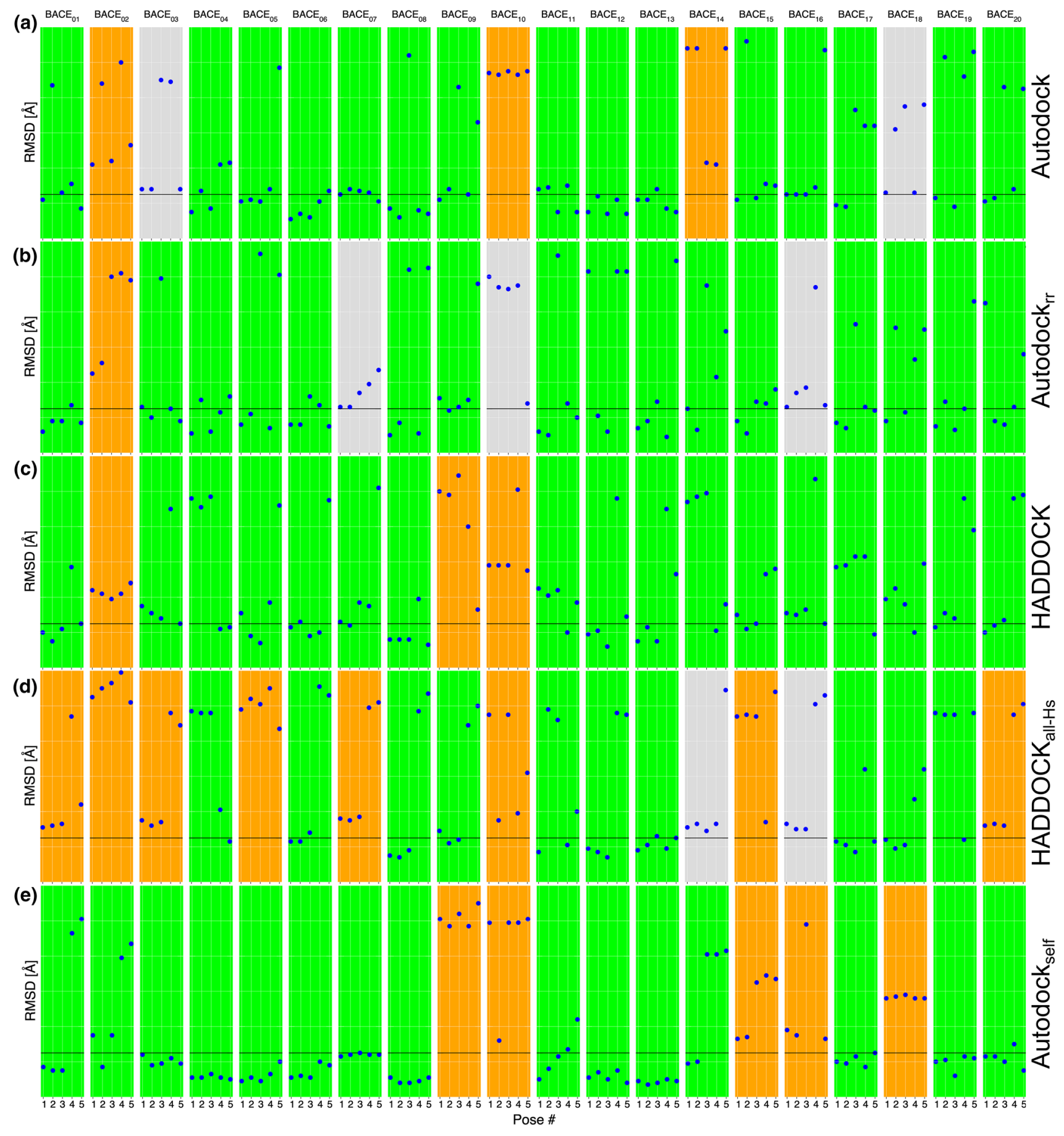

Fig. 3 Performance of the Autodock (a), Autodock rr $_{\text {(b), HADDOCK }}$ (c) and $\mathrm{HADDOCK}_{\mathrm{all}-\mathrm{Hs}}$ (d) protocols in reproducing the near-native conformations of the 20 BACE- 1 ligands. Green and grey panels refer to targets for which we obtained at least one pose within the top 5 featuring a value of the ligand $\mathrm{RMSD} \leq 2.5 \AA$ and $\leq 3 \AA$ respectively, while orange boxes indicate cases for which no such poses were found among the top 5 ones

$\mathrm{RoG}_{\mathrm{BS}}$ in the range 9.10-9.44 $\AA$ from the initial value of $9.79 \AA$ found in $1 \mathrm{SGZ}$.

Notably, among the 200 cluster representatives selected for docking calculations and corresponding to values of $\mathrm{RoG}_{\mathrm{BS}}$ in the range 9.10-9.44 $\AA$, a larger fraction derived ing of all ligands (Fig. 1c), corresponding to a decrease of 
Fig. 4 Normalized distributions (bin size $=0.1 \AA$ ) of $\mathrm{RMSD}_{\mathrm{BS}}$ calculated with respect to the 20 experimental structures of ligands in complex with BACE-1 for $\mathrm{MD}_{\text {apo }}$ (upper panel) and $\mathrm{EDES}_{3 \mathrm{w}}$ (middle panel) trajectories, as well as for the ensemble of 200 BACE-1 structures used in ensemble docking calculations (lower panel, see "Materials and Methods" for details on the cluster analysis). The insets in the upper and middle panels represent enlargements of the left-hand region of the corresponding graphs

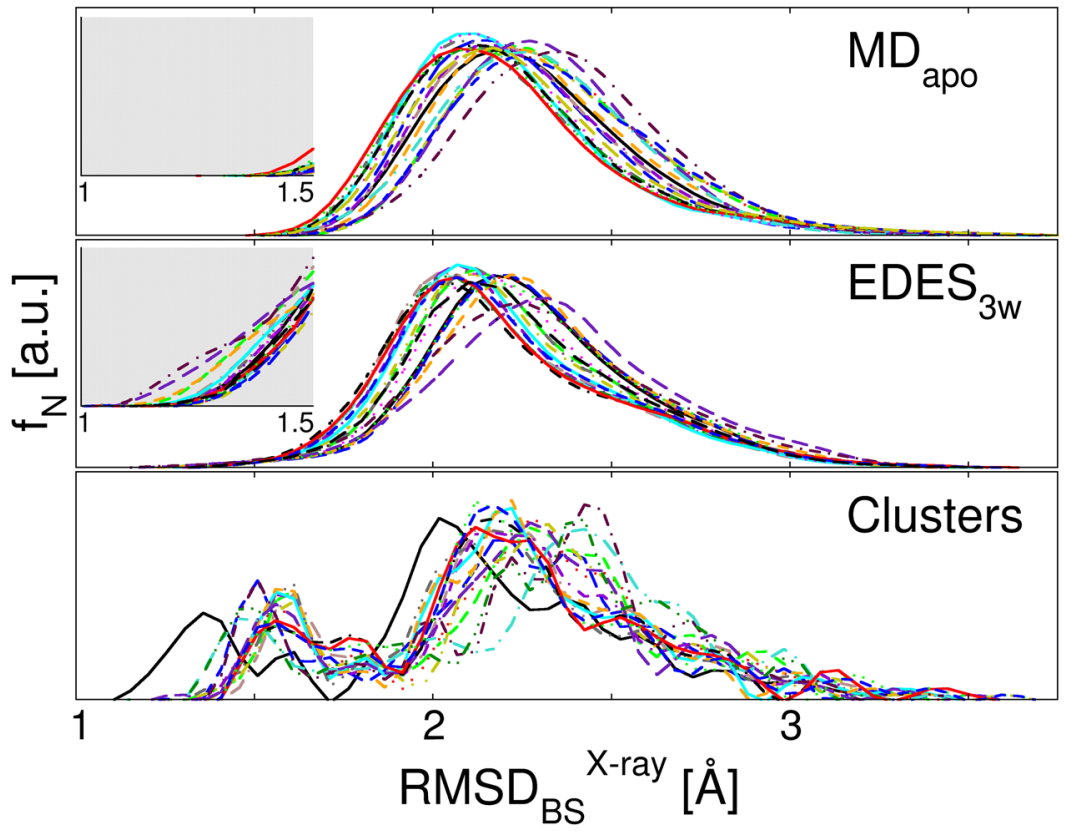

from $\mathrm{EDES}_{3 \mathrm{w}}$ (Fig. S1). In addition, there are several structures corresponding to $\mathrm{RoG}_{\mathrm{BS}}$ values close to or even larger than $9.79 \AA$. Thus, while improving the sampling of relatively closed protein conformations, our protocol still allows for sampling of open ones. Moreover, our multi-step cluster analysis confirmed its tendency to select a large (even larger than that sampled along the MD trajectories) fraction of low$\mathrm{RMSD}_{\mathrm{BS}}$ geometries with respect to all the experimental reference structures (Fig. 4 and Table 3). It is worth pointing out that for all targets we obtained some conformations of the binding site that are virtually identical to the experimental structures, as testified by the lowest $\mathrm{RMSD}_{\mathrm{BS}}$ values, all around $1 \AA$ (Table 3).

We also evaluated the performance of our method in generating druggable conformations of BACE-1 [44]. Table 4 shows the results of this analysis on the 200 receptor conformations used for ensemble-docking calculations, compared with the values obtained for the targets investigated in [13]. It clearly appears that also the approach used in this work can generate a consistently relevant fraction of structures associated with a large druggability score $D$.

\section{Generation of near-native ligand conformers}

The performance of our template-based similarity protocol in generating near-native conformations of the 20 BACE-1 ligands is summarized in Table 3. This Table reports the statistics of the RMSD calculated on heavy atoms of each ligand after structural alignment on the reference conformation extracted from the experimental structure of the corresponding complex (hereafter indicated as RMSD $\mathrm{R}_{\text {lig-fit, }}$, to be distinguished from the same value calculated in the complex after alignment of the protein interface region). In all cases the minimum RMSD $_{\text {lig-fit }}$ values are lower than $2 \AA$, confirming the accuracy of the approach reported in [16] in reproducing at least one near-native conformation of all the macrocycle ligands considered in this work. Overall, these values are slightly larger than those obtained for the sampling of holo-like conformations of the receptor (2nd and 3rd columns in Table 3). Moreover, in 4 (1) out of 20 cases we obtained an average $\operatorname{RMSD}_{\text {lig-fit }}>2(2.5) \AA$, and in 6 out of 20 cases we obtained values of $\mathrm{RMSD}_{\text {lig - fit }}^{\min }>1.5 \AA$, and these ligands are (except for $\mathrm{BACE}_{03}$ and $\mathrm{BACE}_{15}$ ) exactly those for which we obtained the less accurate docking results. Note that, in virtually all cases the value of $\mathrm{RMSD}_{\text {lig-fit }}$ reported in Table 3 for the 10 conformers used in docking calculations is very marginally larger than that calculated over the up to 500 conformers generated with OpenEye OMEGA (the only exception being $\mathrm{BACE}_{18}$, see Fig. S2). Therefore, the issue with the generation of the "correct" conformers of the macrocyclic ligands considered here is not related to the selection of a few representative conformers, but to the sampling process itself, as already documented by others [48-50]. A closer look to Fig. S2 reveals that a large fraction of the 10 conformers displayed $\mathrm{RMSD}_{\text {lig-fit }}$ values lower than $1.5 \AA$ only for $\mathrm{BACE}_{01}, \mathrm{BACE}_{03}, \mathrm{BACE}_{04}$, $\mathrm{BACE}_{05}, \mathrm{BACE}_{06}, \mathrm{BACE}_{08}, \mathrm{BACE}_{11}, \mathrm{BACE}_{12}, \mathrm{BACE}_{13}$, $\mathrm{BACE}_{17}$ and $\mathrm{BACE}{ }_{19}$, which grossly correspond to the systems for which both the most successful approaches (Autodock ${ }_{r r}$ and HADDOCK) were able to find near-native binding poses (Table 2). In contrast, ligands such as $\mathrm{BACE}_{02}, \mathrm{BACE}_{07}, \mathrm{BACE}_{10}, \mathrm{BACE}_{14}, \mathrm{BACE}_{15}$ and $\mathrm{BACE}_{16}$ 
Table 3 Performances of our methodology evaluated separately for the generation of protein and ligand conformations similar to those found in the ligand/BACE-1 experimental structures

\begin{tabular}{|c|c|c|c|}
\hline \multirow[t]{2}{*}{ System } & \multicolumn{2}{|l|}{ Protein } & \multirow{2}{*}{$\begin{array}{l}\text { Ligands } \\
\text { RMSD }_{\text {lig-fit }}[\AA]\end{array}$} \\
\hline & $\mathrm{RMSD}_{\mathrm{BS}}^{\min }[\AA ̊]$ & $\begin{array}{l}\% \\
\operatorname{RMSD}_{\mathrm{BS}}<1.5 \AA\end{array}$ & \\
\hline $\mathrm{BACE}_{01}$ & 1.13 & 16 & $1.11-1.71(1.44 \pm 0.24)$ \\
\hline $\mathrm{BACE}_{02}$ & 1.08 & 15 & $1.16-3.08(2.70 \pm 0.63)$ \\
\hline $\mathrm{BACE}_{03}$ & 1.07 & 15 & $0.95-1.44(1.25 \pm 0.15)$ \\
\hline $\mathrm{BACE}_{04}$ & 1.08 & 19 & $1.03-2.05(1.55 \pm 0.44)$ \\
\hline $\mathrm{BACE}_{05}$ & 1.07 & 15 & $0.82-3.05(1.50 \pm 0.73)$ \\
\hline $\mathrm{BACE}_{06}$ & 1.19 & 15 & $0.58-1.38(0.98 \pm 0.23)$ \\
\hline $\mathrm{BACE}_{07}$ & 1.10 & 17 & $1.62-2.77(2.08 \pm 0.45)$ \\
\hline $\mathrm{BACE}_{08}$ & 1.07 & 17 & $0.57-1.51(0.92 \pm 0.29)$ \\
\hline BACE $_{09}$ & 1.17 & 17 & $1.52-2.38(1.96 \pm 0.38)$ \\
\hline $\mathrm{BACE}_{10}$ & 1.13 & 17 & $1.54-2.33(1.98 \pm 0.33)$ \\
\hline $\mathrm{BACE}_{11}$ & 1.06 & 17 & $1.03-2.24(1.64 \pm 0.48)$ \\
\hline $\mathrm{BACE}_{12}$ & 1.07 & 17 & $0.68-1.79(1.13 \pm 0.44)$ \\
\hline $\mathrm{BACE}_{13}$ & 1.06 & 16 & $0.52-1.00(0.75 \pm 0.16)$ \\
\hline $\mathrm{BACE}_{14}$ & 0.98 & 16 & $1.37-3.34(2.48 \pm 0.81)$ \\
\hline $\mathrm{BACE}_{15}$ & 1.16 & 14 & $1.53-2.23(1.95 \pm 0.33)$ \\
\hline $\mathrm{BACE}_{16}$ & 1.17 & 14 & $1.83-3.53(2.39 \pm 0.59)$ \\
\hline $\mathrm{BACE}_{17}$ & 1.13 & 14 & $1.08-1.58(1.34 \pm 0.18)$ \\
\hline $\mathrm{BACE}_{18}$ & 1.14 & 14 & $1.53-2.01(1.81 \pm 0.11)$ \\
\hline $\mathrm{BACE}_{19}$ & 1.07 & 16 & $1.17-1.51(1.33 \pm 0.10)$ \\
\hline $\mathrm{BACE}_{20}$ & 1.20 & 15 & $1.49-3.26(1.83 \pm 0.52)$ \\
\hline
\end{tabular}

The 2nd column reports the lowest $\mathrm{RMSD}_{\mathrm{BS}}$ calculated across the 200 receptor conformations with respect to each experimental structure. The 3rd column reports the percentage of conformations displaying an $\mathrm{RMSD}_{\mathrm{BS}}$ lower than $1.5 \AA$. The last column reports the minimum and maximum RMSD values (calculated on the nonhydrogenous atoms with respect to the structure of each ligand in the experimental structure), as well as the average and standard deviation within parentheses. Values of $\mathrm{RMSD}_{\mathrm{lig}-\mathrm{fit}}^{\mathrm{min}}$ larger than $1.5 \AA$ and average values of $\mathrm{RMSD}_{\text {lig-fit }}$ larger than $2 \mathrm{~A}$ are italicized and bolded, respectively

Table 4 Performance of our approach in generating druggable conformations of the binding site

\begin{tabular}{lrrrrr}
\hline $\begin{array}{l}\text { \% structures with } D \\
\text { greater than }\end{array}$ & 0.5 & 0.6 & 0.7 & 0.8 & 0.9 \\
\hline BACE-1 & 12.5 & 8.0 & 6.5 & 2.5 & 1.0 \\
BGT* & 15.8 & 10.2 & 6.8 & 4.6 & 1.4 \\
RIC* & 3.2 & 2.4 & 1.6 & 0.4 & 0.2 \\
ABP* & 7.6 & 5.2 & 3.0 & 1.8 & 0.4 \\
\hline
\end{tabular}

The percentages of structures featuring druggability scores $D$ larger than $0.5-0.9$ are reported in columns $2-6$, respectively. The corresponding values for the benchmark systems considered in the original EDES publication are reported for comparison

$B G T$ T4 phage $\beta$-glucosyltransferase, $R I C$ recombinant ricin, $A B P$ allose binding protein

*From Ref. [13] featured most conformations significantly distorted from the structure of the ligand in complex with BACE- 1 . This analysis further confirms previous findings on the importance of sampling not only the correct conformations of the protein but also that of the ligands, as well as the exponential impact on accuracy arising from the combination of even minimal displacements from the correct structures in both partners [16].

\section{Stage 1b}

This is a self-docking stage at which the participants could employ the bound protein structures-but not those of the compounds-released by the organizers. The comparison between the data obtained at stages $1 \mathrm{a}$ and $1 \mathrm{~b}$ is instructive to evaluate the performance of the docking protocol in the presence of the correct structures of the receptor. This enables to highlight drawbacks likely unrelated to the protein flexibility problem, as well as to confirm the importance of using a relevant fraction of correct conformers of the ligand in ensemble-docking calculations. We performed this exercise using AutoDock; namely, we docked, for each ligand, the 10 conformers used in stage 1a on the corresponding experimental structure of the receptor.

Table 5 Summary of the self-docking results obtained with the Auto$d_{\text {ock }} k_{\text {self }}$ protocol for each of the 20 BACE-1 ligands

\begin{tabular}{llcr}
\hline Target ligand & RMSD $^{\text {min }}$ & RMSD $^{1}$ & $\langle$ RMSD $\rangle$ \\
\hline BACE $_{01}$ & 1.5 & 1.7 & $4.8 \pm 4.5$ \\
BACE $_{02}$ & 1.7 & 3.5 & $5.1 \pm 3.1$ \\
BACE $_{03}$ & 1.8 & 2.4 & $2.0 \pm 0.3$ \\
BACE $_{04}$ & 1.0 & 1.1 & $1.1 \pm 0.1$ \\
BACE $_{05}$ & 0.9 & 0.9 & $1.2 \pm 0.5$ \\
BACE $_{06}$ & 1.1 & 1.1 & $1.4 \pm 0.4$ \\
BACE $_{07}$ & 2.3 & 2.3 & $2.4 \pm 0.1$ \\
BACE $_{08}$ & 0.8 & 1.1 & $0.9 \pm 0.2$ \\
BACE $_{09}$ & $\mathbf{9 . 7}$ & 10.1 & $10.2 \pm 0.5$ \\
BACE $_{10}$ & $\mathbf{3 . 2}$ & 9.9 & $8.6 \pm 3.0$ \\
BACE $_{11}$ & 1.0 & 1.0 & $2.4 \pm 1.3$ \\
BACE $_{12}$ & 0.8 & 1.1 & $1.2 \pm 0.3$ \\
BACE $_{13}$ & 0.7 & 0.9 & $0.9 \pm 0.1$ \\
BACE $_{14}$ & 1.9 & 1.9 & $5.7 \pm 3.4$ \\
BACE $_{15}$ & $\mathbf{3 . 3}$ & 3.3 & $5.4 \pm 1.8$ \\
BACE $_{16}$ & $\mathbf{3 . 3}$ & 3.8 & $6.6 \pm 4.2$ \\
BACE $_{17}$ & 1.7 & 2.0 & $2.1 \pm 0.3$ \\
BACE $_{18}$ & $\mathbf{5 . 6}$ & 5.6 & $5.7 \pm 0.1$ \\
BACE $_{19}$ & 1.2 & 2.0 & $2.0 \pm 0.4$ \\
BACE $_{20}$ & 1.5 & 2.3 & $2.2 \pm 0.5$ \\
\hline${ }$ & & &
\end{tabular}

All values are expressed in $\AA$. $\mathrm{RMSD}^{\text {min }}$ values larger than $2.5 \AA$ are bolded 
Table 5 reports the overall performance of Autodock self, while Fig. 3e shows a more detailed analysis of the top 5 poses for each BACE-1 ligand. Interestingly, a marginal improvement was seen with respect to the results obtained with the Autodock protocol at stage 1a, while the success rate evaluated as the number of ligands for which at least one pose featured a value of the RMSD $\leq 2.5 \AA$ remained $75 \%$. This result was in part expected, in view of the good sampling of holo-like protein conformations obtained with our modified EDES protocol (Table 3). Moreover, also in this case the most challenging ligands include those featuring the largest values of RMSD lig-fit $_{\text {and/or RMSD }}^{\text {min }}$ in in Table 3. In particular, wrong poses were found for $\mathrm{BACE}_{09}, \mathrm{BACE}_{10}$, $\mathrm{BACE}_{15}, \mathrm{BACE}_{16}$, and $\mathrm{BACE}_{18}$, while for $\mathrm{BACE}_{07}$ all poses have RMSD values close to $2.5 \AA$. While further relaxation and rescoring of these poses is expected to increase the success rate, we note that a very minor conformational change towards the correct geometry of the binding site was sufficient to find at least one pose with RMSD lower than $2 \AA$ for $\mathrm{BACE}_{02}$.

\section{Conclusions and perspectives}

We report the performance of our hybrid ensemble-docking approach in its first participation to a D3R Grand Challenge competition. The approach is founded on a template-based algorithm to select proper ligand conformers, and on our recently published EDES protocol (implemented here with small modifications with respect to the original version) to sample holo-like protein conformations starting from the apo one. With regard to the generation of holo-like protein conformations, EDES was able to sample near-native BACE-1 conformations (particularly at the binding site) for all of the 20 complexes formed between the protein and the congeneric ligands subject of this study. This points to the general applicability of this approach in sampling holo-like conformations for systems exploiting different kinds of rearrangements upon ligand binding, including minor flap movements such as in the case of BACE-1. A very good accuracy in reproducing near-native ligand conformers was achieved also by our template-based approach. These performances reflected in the relatively high accuracy in the prediction of near-native binding poses. Independently of the docking program used, our method was able to find near-native poses among the top 5 ones for at least $75 \%$ of the 20 complexes selected for the pose prediction sub-challenge. While HADDOCK found near-native poses for more targets than AutoDock, the latter featured the best overall performance when coupled to a computationally cheap post-docking relaxation of the poses. Performing docking calculations on the apo experimental structure of BACE-1 resulted in significantly less accurate predictions, due to the unaccounted rearrangements of the protein flap occurring upon ligand binding. Finally, the good performance of our approach was testified by the only slight overall improvement obtained when performing self-docking calculations on the 20 experimental holo receptor structures.

Note that in this Grand Challenge we also submitted pose predictions following the template-based protocol described in [16] $\left(H A D D O C K_{\mathrm{tb}}\right)$ in which an ideal choice of both ligand and receptor conformations is made based on ligand similarity to known PDB entries. As in GC3, this approach led to an excellent performance with $\left\langle\mathrm{RMSD}^{\mathrm{min}}\right\rangle,\left\langle\mathrm{RMSD}^{1}\right\rangle$ and $\langle$ RMSD $\rangle$ respectively of $1.4 \AA, 1.66 \AA$ and $1.95 \AA$, which demonstrates that a template-based approach remains the best strategy when 3D structures of related complexes are available in the PDB. However, when this is not the case, our EDES approach appears to be an attractive alternative. Moreover, it has been proposed that including MD-generated receptor conformations (such as those obtained from EDES) in the virtual screening protocol could promote the discover of new active chemotypes, especially for flexible receptors, in which entirely new pocket conformations may be revealed for potential ligand binding [51-53].

Further developments of the method will include improved identification of putative binding sites and of their key residues, coupling of EDES with the use of co-solvents, and exploitation of different cluster methodologies.

Acknowledgements A.B. gratefully acknowledges the Sardinia Regional Government for the financial support of his Ph.D. scholarship (P.O.R. Sardegna F.SE., Operational Programme of the Autonomous Region of Sardinia, European Social Fund 2014-2020-Axis III Education and Training, Thematic Goal 10, Priority of Investment 10ii, Specific Goal 10.5., Action Partnership Agreement 10.5.12). This work was done as part of the BioExcel CoE (www.bioexcel.eu), a project funded by the European Union Horizon 2020 Program under Grant Agreements 675728 and 823830 (to A. M. J. J. B.) with financial support from the Dutch Foundation for Scientific Research (NWO) (TOPPUNT Grant 718.015.001.

\section{References}

1. Gaieb Z, Parks CD, Chiu M et al (2019) D3R Grand Challenge 3: blind prediction of protein-ligand poses and affinity rankings. $\mathrm{J}$ Comput Aided Mol Des 33:1-18. https://doi.org/10.1007/s1082 2-018-0180-4

2. Gaieb Z, Liu S, Gathiaka S et al (2018) D3R Grand Challenge 2: blind prediction of protein-ligand poses, affinity rankings, and relative binding free energies. J Comput Aided Mol Des 32:1-20. https://doi.org/10.1007/s10822-017-0088-4

3. Gathiaka S, Liu S, Chiu M et al (2016) D3R grand challenge 2015: evaluation of protein-ligand pose and affinity predictions. J Comput Aided Mol Des 30:651-668. https://doi.org/10.1007/ s10822-016-9946-8

4. Venugopal C, Demos C, Jagannatha Rao K et al (2008) Betasecretase: structure, function, and evolution. CNS Neurol Disord 7:278-294. https://doi.org/10.2174/187152708784936626 
5. Cole SL, Vassar R (2007) The Alzheimer's disease betasecretase enzyme, BACE1. Mol Neurodegener 2:22. https://doi. org/10.1186/1750-1326-2-22

6. Nalivaeva NN, Turner AJ (2013) The amyloid precursor protein: a biochemical enigma in brain development, function and disease. FEBS Lett 587:2046-2054. https://doi.org/10.1016/j.febs1 et.2013.05.010

7. Murphy MP, LeVine H (2010) Alzheimer's disease and the amyloid- $\beta$ peptide. J Alzheimers Dis 19:311-323. https://doi. org/10.3233/JAD-2010-1221

8. Berman HM, Westbrook J, Feng Z et al (2000) The Protein Data Bank. Nucleic Acids Res 28:235-242. https://doi.org/10.1093/ nar/28.1.235

9. Prati F, Bottegoni G, Bolognesi ML, Cavalli A (2018) BACE-1 inhibitors: from recent single-target molecules to multitarget compounds for Alzheimer's disease: miniperspective. J Med Chem 61:619-637. https://doi.org/10.1021/acs.jmedchem.7b00393

10. Moussa CE-H (2017) Beta-secretase inhibitors in phase I and phase II clinical trials for Alzheimer's disease. Expert Opin Investig Drugs 26:1131-1136. https://doi.org/10.1080/13543 784.2017.1369527

11. Amaro RE, Baudry J, Chodera J et al (2018) Ensemble docking in drug discovery. Biophys J 114:2271-2278. https://doi. org/10.1016/j.bpj.2018.02.038

12. Huang S-Y, Zou X (2007) Ensemble docking of multiple protein structures: considering protein structural variations in molecular docking. Proteins Struct Funct Bioinform 66:399-421. https://doi. org/10.1002/prot.21214

13. Basciu A, Malloci G, Pietrucci F et al (2019) Holo-like and druggable protein conformations from enhanced sampling of binding pocket volume and shape. J Chem Inf Model 59:1515-1528. https ://doi.org/10.1021/acs.jcim.8b00730

14. Zhao H, Caflisch A (2015) Molecular dynamics in drug design. Eur J Med Chem 91:4-14. https://doi.org/10.1016/j.ejmec h.2014.08.004

15. De Vivo M, Masetti M, Bottegoni G, Cavalli A (2016) Role of molecular dynamics and related methods in drug discovery. J Med Chem 59:4035-4061. https://doi.org/10.1021/acs.jmedc hem. 5 b01684

16. Koukos PI, Xue LC, Bonvin AMJJ (2019) Protein-ligand pose and affinity prediction: lessons from D3R Grand Challenge 3. J Comput Aided Mol Des 33:83-91. https://doi.org/10.1007/s1082 2-018-0148-4

17. Hong L, Tang $\mathbf{J}$ (2004) Flap position of free memapsin 2 ( $\beta$-secretase), a model for flap opening in aspartic protease catalysis. Biochemistry 43:4689-4695. https://doi.org/10.1021/bi049 8252

18. Laio A, Parrinello M (2002) Escaping free-energy minima. Proc Natl Acad Sci USA 99:12562-12566. https://doi.org/10.1073/ pnas.202427399

19. Hawkins PCD, Skillman AG, Warren GL et al (2010) Conformer generation with OMEGA: algorithm and validation using high quality structures from the protein databank and cambridge structural database. J Chem Inf Model 50:572-584. https://doi. org/10.1021/ci100031x

20. Morris GM, Huey R, Lindstrom W et al (2009) AutoDock4 and AutoDockTools4: automated docking with selective receptor flexibility. J Comput Chem 30:2785-2791.

21. Dominguez C, Boelens R, Bonvin AMJJ (2003) HADDOCK: a protein-protein docking approach based on biochemical or biophysical information. J Am Chem Soc 125:1731-1737. https:// doi.org/10.1021/ja026939x

22. van Zundert GCP, Rodrigues JPGLM, Trellet $M$ et al (2016) The HADDOCK2.2 web server: user-friendly integrative modeling of biomolecular complexes. Comput Resour Mol Biol 428:720-725. https://doi.org/10.1016/j.jmb.2015.09.014
23. Hawkins PCD, Skillman AG, Nicholls A (2007) Comparison of shape-matching and docking as virtual screening tools. J Med Chem 50:74-82. https://doi.org/10.1021/jm0603365

24. Wang Y, Backman TWH, Horan K, Girke T (2013) fmcsR: mismatch tolerant maximum common substructure searching in $\mathrm{R}$. Bioinformatics 29:2792-2794. https://doi.org/10.1093/bioinforma tics/btt475

25. Cao Y, Charisi A, Cheng L-C et al (2008) ChemmineR: a compound mining framework for R. Bioinformatics 24:1733-1734. https://doi.org/10.1093/bioinformatics/btn307

26. Kurkcuoglu Z, Koukos PI, Citro N et al (2018) Performance of HADDOCK and a simple contact-based protein-ligand binding affinity predictor in the D3R Grand Challenge 2. J Comput Aided Mol Des 32:175-185. https://doi.org/10.1007/s10822-017-0049-y

27. Altschul SF, Gish W, Miller W et al (1990) Basic local alignment search tool. J Mol Biol 215:403-410. https://doi.org/10.1016/ S0022-2836(05)80360-2

28. Williams CJ, Headd JJ, Moriarty NW et al (2018) MolProbity: more and better reference data for improved all-atom structure validation: PROTEIN SCIENCE.ORG. Protein Sci 27:293-315. https://doi.org/10.1002/pro.3330

29. Case DA, Ben-Shalom IY, Brozell SR et al (2018) AMBER18. University of California, San Francisco

30. Wang L-P, Martinez TJ, Pande VS (2014) Building force fields: an automatic, systematic, and reproducible approach. J Phys Chem Lett 5:1885-1891. https://doi.org/10.1021/jz500737m

31. Wang L-P, McKiernan KA, Gomes J et al (2017) Building a more predictive protein force field: a systematic and reproducible route to AMBER-FB15. J Phys Chem B 121:4023-4039. https://doi. org/10.1021/acs.jpcb.7b02320

32. Joung IS, Cheatham TE (2008) Determination of alkali and halide monovalent ion parameters for use in explicitly solvated biomolecular simulations. J Phys Chem B 112:9020-9041. https://doi. org/10.1021/jp8001614

33. Hopkins CW, Le Grand S, Walker RC, Roitberg AE (2015) Long-time-step molecular dynamics through hydrogen mass repartitioning. J Chem Theory Comput 11:1864-1874. https:// doi.org/10.1021/ct5010406

34. Barducci A, Bussi G, Parrinello M (2008) Well-tempered metadynamics: a smoothly converging and tunable free-energy method. Phys Rev Lett 100:020603. https://doi.org/10.1103/PhysRevLet t.100.020603

35. Piana S, Laio A (2007) A bias-exchange approach to protein folding. J Phys Chem B 111:4553-4559. https://doi.org/10.1021/jp067 8731

36. Abraham MJ, Murtola T, Schulz R et al (2015) GROMACS: high performance molecular simulations through multi-level parallelism from laptops to supercomputers. SoftwareX. https://doi. org/10.1016/j.softx.2015.06.001

37. Tribello GA, Bonomi M, Branduardi D et al (2014) PLUMED 2: new feathers for an old bird. Comput Phys Commun 185:604-613. https://doi.org/10.1016/j.cpc.2013.09.018

38. Sousa da Silva AW, Vranken WF (2012) ACPYPE - AnteChamber PYthon Parser interfacE. BMC Res Notes 5:367. https://doi. org/10.1186/1756-0500-5-367

39. Hayward S (2004) Identification of specific interactions that drive ligand-induced closure in five enzymes with classic domain movements. J Mol Biol 339:1001-1021. https://doi.org/10.1016/j. jmb.2004.04.004

40. Gerstein M (1998) A database of macromolecular motions. Nucleic Acids Res 26:4280-4290. https://doi.org/10.1093/ nar/26.18.4280

41. van Aalten DMF, Bywater R, Findlay JBC et al (1996) PRODRG, a program for generating molecular topologies and unique molecular descriptors from coordinates of small molecules. J Comput Aided Mol Des 10:255-262. https://doi.org/10.1007/BF00355047 
42. Wang J, Wolf RM, Caldwell JW et al (2004) Development and testing of a general amber force field. J Comput Chem 25:1157-1174

43. Le Guilloux V, Schmidtke P, Tuffery P (2009) Fpocket: an open source platform for ligand pocket detection. BMC Bioinform 10:168. https://doi.org/10.1186/1471-2105-10-168

44. Schmidtke P, Barril X (2010) Understanding and predicting druggability. A High-throughput method for detection of drug binding sites. J Med Chem 53:5858-5867. https://doi.org/10.1021/jm100 $574 \mathrm{~m}$

45. Antunes DA, Devaurs D, Kavraki LE (2015) Understanding the challenges of protein flexibility in drug design. Expert Opin Drug Discov 10:1301-1313. https://doi.org/10.1517/17460 441.2015.1094458

46. Du X, Li Y, Xia Y-L et al (2016) Insights into protein-ligand interactions: mechanisms, models, and methods. Int J Mol Sci 17:144. https://doi.org/10.3390/ijms17020144

47. Buonfiglio R, Recanatini M, Masetti M (2015) Protein flexibility in drug discovery: from theory to computation. ChemMedChem 10:1141-1148. https://doi.org/10.1002/cmdc.201500086

48. Chen I-J, Foloppe N (2013) Tackling the conformational sampling of larger flexible compounds and macrocycles in pharmacology and drug discovery. Bioorg Med Chem 21:7898-7920. https://doi. org/10.1016/j.bmc.2013.10.003
49. Cleves AE, Jain AN (2017) ForceGen 3D structure and conformer generation: from small lead-like molecules to macrocyclic drugs. J Comput Aided Mol Des 31:419-439. https://doi.org/10.1007/ s10822-017-0015-8

50. Jain AN, Cleves AE, Gao Q et al (2019) Complex macrocycle exploration: parallel, heuristic, and constraint-based conformer generation using ForceGen. J Comput Aided Mol Des 33:531558. https://doi.org/10.1007/s10822-019-00203-1

51. Tarcsay Á, Paragi G, Vass M et al (2013) The impact of molecular dynamics sampling on the performance of virtual screening against GPCRs. J Chem Inf Model 53:2990-2999. https://doi. org/10.1021/ci400087b

52. Li YY, An J, Jones SJ (2011) A computational approach to finding novel targets for existing drugs. PLoS Comput Biol 7:e1002139

53. Amaro RE, Li WW (2010) Emerging methods for ensemble-based virtual screening. Curr Top Med Chem 10:3-13

Publisher's Note Springer Nature remains neutral with regard to jurisdictional claims in published maps and institutional affiliations. 